\title{
Procedimientos quirúrgicos de los miembros de la Sociedad Española de Cirugía de la Mano (SECMA) para la artrosis trapecio-metacarpiana: Un examen de las tendencias de la práctica clínica actual
}

\section{Surgical Procedures of Members of the Spanish Society of Surgery of the Hand (SECMA) for Trapezius- Metacarpal Arthrosis: An Examination of Trends in Current Clinical Practice}

\author{
Raquel Cantero-Téllez ${ }^{1,2}$ Jorge Hugo Villafañe ${ }^{3} \quad$ Kristin Valdes $^{4}$ Pedro Berjano ${ }^{5}$
}

${ }^{1}$ Área de Fisioterapia - Facultad de Ciencias de la Salud, Universidad de Málaga, Málaga, España

${ }^{2}$ Centro Tecan, Clínica de la mano, Málaga, España

${ }^{3}$ IRCCS Don Gnocchi Foundation, Milan, Italy

${ }^{4}$ Hand Works Therapy, Gannon University, Florida, United States

${ }^{5}$ IRCCS Instituto Ortopedico Galeazzi, Milan, Italy
Address for correspondence Raquel Cantero-Téllez, PhD, MSc, PT, OT, Área de Fisioterapia - Facultad de Ciencias de la Salud, Universidad de Málaga, Málaga, España (e-mail: cantero@uma.es).

Rev Iberam Cir Mano 2017;45:12-15.

\begin{abstract}
Resumen
Objetivo El objetivo principal de este estudio fue determinar los diferentes enfoques terapéuticos empleados en la práctica clínica habitual entre los cirujanos de mano en España en el abordaje de la artrosis trapecio-metacarpiana (TMC).

Material y Método Se desarrolló una encuesta de 15 ítems para determinar los patrones terapéuticos en la práctica clínica de los cirujanos de mano en España que tratan la artrosis TMC. Esa prueba piloto se distribuyó a través de un servicio de encuestas en línea profesional (SurveyMonkey).

Palabras Claves

- pulgar

- rizartrosis

- encuesta

- trapeciometacarpiana

- cirugía

Resultados El 94\% de los cirujanos de mano en España se basan en la intensidad de dolor referida por el paciente para tomar una decisión quirúrgica. El 75\% los inmoviliza durante un periodo de 3 semanas tras la cirugía y solo el 36\% de los encuestados evalúa la intensidad de dolor después de la cirugía.

Conclusiones Esa encuesta proporciona datos valiosos en relación con los patrones terapéuticos de la práctica clínica actual en el tratamiento quirúrgico de la artrosis TMC en los cirujanos de mano españoles.

Nivel de Evidencia Nivel V.
\end{abstract}

received

December 10, 2016

accepted

March 25, 2017
DOI https://doi.org/

$10.1055 / \mathrm{s}-0037-1602795$.

ISSN 1698-8396.
Copyright $\odot 2017$ by Thieme Revinter

Publicações Ltda, Rio de Janeiro, Brazil
License terms

(c) $(1) \$$ 


\author{
Abstract \\ Keywords \\ - thumb \\ - basal thumb \\ osteoarthritis \\ - survey \\ - carpometa- \\ carpal joint \\ - surgery
}

Purpose The main purpose of this study was to elucidate the current practice patterns among the Spanish hand surgeons in the surgical treatment of the thumb carpometacarpal (CMC) joint osteoarthritis (OA).

Methods A 15-item survey to determine the practice patterns of the Spanish hand surgeons who treat $\mathrm{CMC}$ joint $\mathrm{OA}$ was developed, pilot tested, and distributed through a professional online survey service (SurveyMonkey, Palo Alto, CA) to assure confidentiality and anonymity.

Results $94 \%$ of the Spanish hand surgeons make their surgical decision to perform surgery based on the patients reported pain level. $75 \%$ of the surgeons immobilize their patients between 1 and 3 weeks after surgery and only $36 \%$ of the interviewees assess pain intensity after surgery.

Conclusions This survey provides valuable data regarding the current practice patterns in the surgical treatment of thumb CMC OA by the Spanish hand surgeons. Level of Evidence Level V.

\section{Introducción}

Los pacientes que sufren artrosis TMC (trapecio-metacarpiana) requieren, generalmente, tratamiento especializado por parte de cirujanos de mano.

Están descritas una gran variedad de técnicas para clasificar la enfermedad, determinar si es necesaria o no la cirugía, y realizar el procedimiento quirúrgico adecuado para cada caso. Numerosos estudios llegan a la conclusión de que ningún procedimiento quirúrgico utilizado para la artrosis TMC del pulgar es mejor que otro. ${ }^{1-4}$

Una encuesta realizada a los miembros de la Sociedad Americana de Cirugía de la Mano, demostró que, a pesar de la evidencia reciente que sugiere que ni la reconstrucción de ligamentos ni la ligamentoplastia de suspensión aportan beneficio adicional comparado con la trapecectomía, algunos cirujanos la habían convertido en el procedimiento más utilizado. ${ }^{5}$ Esos resultados donde la evidencia científica no se correspondía con la práctica clínica habitual utilizada por cirujanos de mano, fue la base para la realización de este estudio en la población Española.

El objetivo principal de este estudio es determinar los patrones habituales de actuación entre los cirujanos de la mano en España en el abordaje de la artrosis TMC.

\section{Material y Método}

Se confeccionó una encuesta de 15 ítems para determinar los hábitos en la práctica clínica entre los cirujanos de mano en España que tratan la artrosis TMC mediante una prueba piloto que se desarrolló y distribuyó a través de un servicio de encuestas en línea profesional (SurveyMonkey, Palo Alto, CA), asegurando así la confidencialidad y el anonimato de los participantes. El procedimiento fue aprobado por el Comité Ético de experimentación de la Universidad de Málaga (CEUMA) (Registro CEUMA 45-2016H). Ciento tres médicos $(n=103)$, miembros de la Sociedad Española de Cirugía de la Mano (SECMA), que desarrollan su actividad clínica en diferentes puntos geográficos de España respondieron a la encuesta. Se analizaron los datos demográficos de los participantes, tratamiento de elección y medidas de valoración de resultados entre otros datos.

\section{Resultados}

La -tabla 1 muestra los resultados más concluyentes del estudio expresados en porcentaje.

\section{Participantes}

La mayor parte de los cirujanos de mano encuestados, el 65\% $(n=67)$, desarrolla su actividad en los hospitales públicos. El resto trabaja en mutuas $6 \%(n=6)$, hospitales privados $9 \%$ $(n=9)$, consultas privadas $9 \%(n=9)$ y otros $11 \%(n=12)$. La mayoría de los médicos que respondieron a la encuesta tienen experiencia como cirujanos de mano durante más de 20 años, el 36\% ( $n=37)$, el $29 \%(n=30)$ tiene una experiencia clínica entre 11 y 20 años y el $18 \%(n=18)$ entre 5 y 10 años. Sólo el $17 \%$ tiene una experiencia < de 5 años en cirugía de mano. Respecto al número de pacientes que acude a consulta diagnosticados de artrosis TMC, el 69\% $(n=71)$ respondió que trata entre 6-20 pacientes por mes. El 12\% ( $n=12$ ) ve más de 20 y el $19 \%(n=20)$ menos de 5 pacientes con artrosis TMC al mes. El 91\% de los médicos que respondieron a la encuesta son cirujanos ortopédicos y el $9 \%$ cirujanos plásticos. Solo 19 de los 103 médicos encuestados posee el título de Doctor.

\section{Tratamiento de Elección}

Para determinar el diagnóstico de la artrosis TMC, el $45 \%$ ( $n=46)$ utiliza la RX (clasificación de Eaton), el 41\% $(n=42)$ se basan en la exploración clínica para establecer el diagnóstico y el $14 \%(n=15)$ indica que utiliza otro método. La mayor parte de los cirujanos consultados (94\%; $\mathrm{n}=96$ ) toman como referencia la intensidad de dolor para tomar una decisión quirúrgica, el $8 \%(n=6)$ considera solo la clasificación de Eaton y solo el $1 \%$ tiene en cuenta la edad del paciente. 
Tabla 1 Información general más relevante del cuestionario $(n=103$; Los resultados están expresados en porcentaje total de respuestas por ítem)

\begin{tabular}{|c|c|c|}
\hline \multirow[t]{2}{*}{ Pregunta } & \multicolumn{2}{|l|}{ Participantes } \\
\hline & Respuestas & [n (\%)] \\
\hline \multicolumn{3}{|l|}{ Información General } \\
\hline 1. ¿En qué estructura trabajas? & Hospital público & $67,(65 \%)$ \\
\hline 2. ¿Cuántos años de experiencia tiene como cirujano de mano? & $>20$ & $37,(36.1 \%)$ \\
\hline 3. ¿Cuántos pacientes trata diagnosticados de rizartrosis al mes? & $6-20$ & $71,(69 \%)$ \\
\hline 4. Información del profesional & Traumatólogo & $93,(91 \%)$ \\
\hline \multicolumn{3}{|l|}{ Criterios diagnósticos para decisión quirúrgica } \\
\hline $\begin{array}{l}\text { 5. ¿Qué criterios considera para el diagnóstico de la Artrosis de la base del } \\
\text { pulgar? }\end{array}$ & Hallazgos rx. de Eaton & $46,(45 \%)$ \\
\hline $\begin{array}{l}\text { 6. Cuando explora a un paciente, ¿Qué factor considera más importante } \\
\text { para optar por una decisión quirúrgica? }\end{array}$ & Intensidad de DOLOR & $96,(94.1 \%)$ \\
\hline $\begin{array}{l}\text { 7. En el caso de tomar como referencia la clasificación de Eaton, ¿Cuándo } \\
\text { considera oportuna la intervención quirúrgica? }\end{array}$ & No la tomo como referencia & $52,(51.2 \%)$ \\
\hline \multicolumn{3}{|l|}{ Técnica quirúrgica } \\
\hline $\begin{array}{l}\text { 8. En los últimos } 3 \text { meses, ¿Qué número de pacientes aproximado ha } \\
\text { tratado quirúrgicamente con dolor en la base del pulgar? }\end{array}$ & $0-25$ & $63,(79.8 \%)$ \\
\hline 9. ¿Qué técnica quirúrgica emplea con mayor frecuencia? & Artroplastia en suspensión & $35,(34 \%)$ \\
\hline \multicolumn{3}{|l|}{ Abordaje post cirugía } \\
\hline $\begin{array}{l}\text { 10. A la hora de determinar la técnica quirúrgica a emplear, ¿Qué criterio } \\
\text { considera? }\end{array}$ & Edad del paciente & $51,(49 \%)$ \\
\hline 11. ¿Qué tipo de inmovilización emplea tras la intervención quirúrgica? & Inmovilización muñeca y pulgar & $84,(82 \%)$ \\
\hline $\begin{array}{l}\text { 12. ¿Cuánto suele ser la duración total de inmovilización post-quirúrgica en } \\
\text { ese tipo de pacientes? }\end{array}$ & 3 semanas & $77,(75 \%)$ \\
\hline 13. ¿Consideras la funcionalidad tras la cirugía? & Sí & $86(84 \%)$ \\
\hline 14. ¿Qué evalúas en el post-operatorio como medida outcome? & Intensidad de dolor & $37,(36 \%)$ \\
\hline 15. ¿Evalúas la oposición del Pulgar tras la cirugía? ¿Qué método utilizas? & Escala de op. Kapandji & 79, (77\%) \\
\hline
\end{tabular}

Más de la mitad de los encuestados $(n=52)$ no consideran la clasificación de Eaton antes de tomar la decisión quirúrgica. El resto, $(n=51)$ considera la cirugía cuando el paciente tiene una clasificación Eaton de 2 o mayor. Los procedimientos quirúrgicos realizados presentan una gran variabilidad con un $34 \%$ de los encuestados $(n=35)$ que utilizan la artroplastia en suspensión, frente a un 30\% $(n=31)$ que lleva a cabo una trapecectomía más ligamentoplastia BurtonPellegrini, siendo esas las dos técnicas quirúrgicas de elección. Las técnicas quirúrgicas menos frecuentes son la utilización de prótesis (11\%), la técnica de Brunelli (2\%) y la artrodesis utilizada en minoría por el $1 \%$. El 22\%, (casi uno de cada 4 entrevistados), informa que emplea otra técnica quirúrgica no descrita con anterioridad (trapecectomía + artroplastia, trapecectomia y estabilización con mini tightrope, Trapezectomia + Weilby modificado, resección parcial artroscópica y mini tight-rope, técnica de Scheker,...).

Entre los factores que influyen a la hora de determinar la técnica quirúrgica a emplear, la edad del paciente es considerada en casi la mitad de los encuestados ( $49 \% ; \mathrm{n}=51)$, el $20 \%(n=20)$ considera la clasificación de Eaton, el 9\% $(n=9)$ la intensidad de dolor referida por el paciente, y $6 \%(n=7)$ la deformidad del pulgar. El 16\% $(n=16)$ reconoce utilizar una técnica quirúrgica que ellos consideran de elección sin tener en cuenta los factores descritos con anterioridad, es decir, utilizan la técnica quirúrgica que aprendieron en su momento o aquella con la que se encuentran más cómodos sin considerar el grado de dolor o la deformidad.

Este estudio no ha encontrado una correlación significativa entre la técnica quirúrgica empleada y el lugar de trabajo donde el cirujano de mano desempeña su labor (sistema público, privado o de mutua) $(p=0.21)$ ( - Tabla 2). La técnica quirúrgica empleada tampoco se corresponde con los años de experiencia del cirujano $(p=0.17)$.

\section{Abordaje Post-quirúrgico y Medidas de Resultados}

Una vez realizada la intervención quirúrgica, el $82 \%(n=84)$ tiende a inmovilizar el pulgar y la muñeca, dejando libre la articulación interfalángica (IF) del pulgar, el 10\% $(n=11)$ no incluye la articulación de la muñeca en la inmovilización post-quirúrgica y el $5 \%(n=5)$ inmoviliza la muñeca y el pulgar incluyendo la IF. Sólo el $2 \%$ de los encuestados $(n=2)$ no emplea inmovilización alguna tras la cirugía y el $1 \%$ refiere utilizar una técnica de inmovilización diferente a las citadas anteriormente. La duración del período de inmovilización varía entre $1-3$ semanas $(75 \% ; n=77)$. El $25 \%(n=26)$ 
Tabla 2 Correlación entre la elección de la técnica quirúrgica y las características del cirujano (Lugar de trabajo y años de experiencia)

\begin{tabular}{|l|l|l|l|}
\hline & Correlación de Pearson & $\begin{array}{l}\text { Estructura } \\
\text { (Lugar de trabajo) }\end{array}$ & $\begin{array}{l}\text { Experiencia } \\
\text { Técnica quirúrgica que emplea con mayor frecuencia }\end{array}$ \\
\cline { 2 - 4 } & $\mathrm{R}$ & 0.12 & 0.19 \\
\cline { 2 - 4 } & $\mathrm{P}$ & 0.21 & 0.17 \\
\hline
\end{tabular}

inmoviliza por un periodo superior a las 3 semanas. La mayoría de los cirujanos evalúan los resultados funcionales después de la cirugía ( $84 \% ; n=86)$. Entre las medidas de evaluación post-quirúrgicas utilizadas nos encontramos la intensidad de dolor empleada por el $36 \%(n=37)$, la fuerza de pinza (29\%; $n=30)$, el recorrido articular $(\mathrm{ROM})$ de la muñeca y el pulgar $11 \%(n=11)$ y la fuerza de prensión $7 \%$ $(n=7)$. El $11 \%(n=12)$ informan que utilizan otras medidas de resultado y el $6 \%(n=6)$ no usa ninguna medida para valorar los resultados. Para evaluar la ROM del pulgar, el 77\% $(n=79)$ utiliza el método de Kapandji.

\section{Discusión}

El objetivo principal de este estudio fue recopilar información sobre los procedimientos de actuación en la práctica clínica habitual entre los cirujanos de mano en España en el abordaje de la artrosis TMC del pulgar. En nuestro estudio encontramos resultados similares a los descritos por Brunton y Wilgis en la Sociedad Americana de Cirugía de la Mano. ${ }^{5} \mathrm{~A}$ pesar de la evidencia aportada en estudios recientes que sugiere que ninguna técnica aporta beneficio adicional sobre la trapezectomía en el tratamiento quirúrgico de la artrosis TMC del pulgar, 6,7 algunos cirujanos las utilizan como procedimiento de elección sin considerar la evidencia científica publicada al respecto. Con respecto al tiempo de inmovilización post-quirúrgica más del $75 \%$ de los cirujanos inmovilizan el pulgar durante 1-3 semanas al igual que en estudios previos. ${ }^{5}$ En lo que se refiere a la valoración de resultados, el $84 \%$ de los cirujanos evalúan los resultados funcionales después de la cirugía. Esto es importante, ya que los objetivos finales de la cirugía de la artrosis TMC es restaurar la función del pulgar. ${ }^{8,9}$ Es llamativo sin embargo, que pese a que existe una relación entre la funcionalidad y la intensidad de dolor, ${ }^{6}$ solo el 36\% valora la intensidad del dolor tras la cirugía cuando la mayor parte de ellos, concretamente el 94\%, toman como referencia la intensidad de dolor referida por el paciente antes de tomar una decisión quirúrgica. Eso impide que se puedan valorar los resultados de forma concreta entre las diferentes técnicas quirúrgicas ya que no existe una concordancia entre las medidas de resultados tomadas como referencia antes de la cirugía y después de la misma.

\section{Conclusiones}

Esta encuesta proporciona datos valiosos en relación a los patrones de la práctica clínica actual en el abordaje quirúrgico de la artrosis TMC del pulgar utilizada por los cirujanos de mano en España. Sería interesante estudiar por qué tanto en España como en otros países, se opta por una u otra técnica quirúrgica más por las preferencias personales que por la evidencia científica. Futuros estudios deberán desarrollarse para poder establecer unas pautas de valoración y tratamiento acordes a cada paciente que permitan elaborar unas guías de práctica clínica para el abordaje quirúrgico de la artrosis TMC. Para ello, es necesario que en la práctica clínica habitual se valoren los mismos parámetros antes y después de la intervención quirúrgica y se recojan de forma sistemática, los datos que nos permitirán comparar los resultados.

Conflicto de Intereses

No se declaran conflictos de intereses.

Agradecimientos

Agradecemos a la Sociedad Española de Cirugía de Mano y especialmente al Dr. Angel Ferreres, por su incondicional ayuda en la elaboración y difusión de las encuestas.

\section{Bibliografía}

1 Villafañe JH, Valdes K, Bissolotti L. Thumb carpometacarpal osteoarthritis: are we closer to gold standards? Eur J Phys Rehabil Med 2016;52(06):907-908

2 Wajon A, Ada L, Edmunds I. Surgery for thumb (trapeziometacarpal joint) osteoarthritis. Cochrane Database Syst Rev 2005;(04):CD004631

3 Martou G, Veltri K, Thoma A. Surgical treatment of osteoarthritis of the carpometacarpal joint of the thumb: a systematic review. Plast Reconstr Surg 2004;114(02):421-432

4 Shuler MS, Luria S, Trumble TE. Basal joint arthritis of the thumb. J Am Acad Orthop Surg 2008;16(07):418-423

5 Brunton LM, Wilgis EF. A survey to determine current practice patterns in the surgical treatment of advanced thumb carpometacarpal osteoarthrosis. Hand (NY) 2010;5(04):415-422

6 Lenoir H, Erbland A, Lumens D, Coulet B, Chammas M. Trapeziectomy and ligament reconstruction tendon interposition after failed trapeziometacarpal joint replacement. Hand Surg Rehabil 2016;35(01):21-26

7 Colegate-Stone TJ, Garg S, Subramanian A, Mani GV. Outcome analysis of trapezectomy with and without pyrocarbon interposition to treat primary arthrosis of the trapeziometacarpal joint. Hand Surg 2011;16(01):49-54

8 Cantero-Téllez R, Martín-Valero R, Cuesta-Vargas A. Effect of muscle strength and pain on hand function in patients with trapeziometacarpal osteoarthritis. A cross-sectional study. Reumatol Clin 2015;11(06):340-344

9 Arazpour M, Soflaei M, Ahmadi Bani M, et al. The effect of thumb splinting on thenar muscles atrophy, pain, and function in subjects with thumb carpometacarpal joint osteoarthritis. Prosthet Orthot Int 2016:0309364616664149 